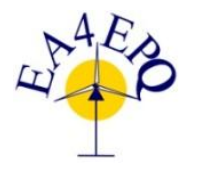

International Conference on Renewable Energies and Power Quality (ICREPQ’13)

Bilbao (Spain), $20^{\text {th }}$ to $22^{\text {th }}$ March, 2013

Renewrable Eenergy, and $\mathscr{P}$ ourer Qualiily. Yournal (RE\&PQJ)

ISSN 2172-038 X, No.11, March 2013

\title{
Production of Biodiesel from WVO Using Small Scale Continuous Ultrasonic Processor
}

\author{
Justin Wood $^{1}$, Jared Slayton ${ }^{1}$, Seth Parrott ${ }^{1}$, Chinyere Mbachu ${ }^{2}$, and Ahmed EISawy ${ }^{3}$ \\ ${ }^{1}$ Undergraduate Students, ${ }^{2} \mathrm{Ph} . \mathrm{D}$ Candidate, and ${ }^{3}$ Professor and Faculty Advisor \\ Department of Manufacturing and Engineering Technology, College of Engineering, Tennessee \\ Technological University, Cookeville, Tennessee 38505-0001, USA \\ Phone \# 0019313723238/Fax \# 0019313723813, emails: ${ }^{1}$ jtwoo42@ @ students.tntech.edu,

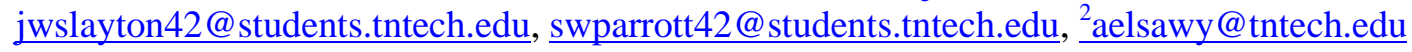

\begin{abstract}
There is a need in the USA to decrease dependency on fossil fuels. One alternative fuel that has gained much popularity in the past few years is biodiesel. Biodiesel can be produced using vegetable oil, waste vegetable oil (WVO), animal fat and yellow grease as raw materials. However, the process of converting a batch of WVO into usable biodiesel is time consuming, requires a human operator to run the system, and necessitates the performance of a chemical titration for each batch of biodiesel produced.

In the first phase of this project, the processor was designed and built by the senior design students utilizing a programmable logic controller (PLC) in conjunction with pumps, valves, temperature sensors, etc. to completely handle the production of biodiesel with minimum operator interaction. This was the first step toward continuous flow processor and the elimination of the titration process. In the second phase of this project, the students integrated a small Hielscher Ultrasound continuous processing unit to the automated system. This paper presents the newly developed system and demonstrates the design aspects of the automated biodiesel production processor using a PLC and ultrasonication (continuous processing) as well as how the chemical titration procedure for each batch is eliminated.
\end{abstract}

\section{Keywords}

Biodiesel, Waste Vegetable Oil, Alternative Energy, Programmable Logic Controllers, Ultrasonication.

\section{Introduction}

With our desire to recycle and reuse solid waste, many people are looking for alternative ways to power vehicles and equipment using biofuel. One alternative fuel for diesel engines is biodiesel. The primary ingredient, waste vegetable oil (WVO), is available and inexpensive. However, the current processors available to produce batch biodiesel are time consuming. They require an operator to run and monitor the system, and necessitate a chemical titration procedure for each batch to be produced. In order to reduce the processing time, a more advanced system is needed. Therefore, the main objective of this project is to explore potential alternatives to the current biodiesel production methods, thus contributing to biodiesel commercialization. This main objective of this project is to develop a small scale continuous biodiesel process through the reduction of the operator interaction time, eliminating the titration process, moving the fluid 
throughout the system using solenoid valves and PLC, use of ultrasonic reactor and presenting the end user with biodiesel upon completion of a full cycle.

\section{Objectives}

The ultimate goal of this project is to significantly increase the production capacity and product quality while reducing cost and human interaction. Therefore, this project is divided into two phases:

a. In the first phase [1], an automated continuous flow system that limits the operator interaction to depositing waste vegetable oil into the processor. The system should automatically take care of moving fluids throughout the system, all chemical reactions, draining of waste glycerol byproduct, and upon completion of a full cycle the system should present the operator with finished biodiesel that meets ASTM standards.

b. In the second phase, a small Hielscher industrial ultrasonic processor UIP500$500 \mathrm{~W}$ continuous biodiesel processor was integrated to the system. This technology creates nano-sized vacuum bubbles (i.e. cavitation) that help to overcome the cohesion and adhesion of the liquid it is mixing [2]. This introduction of cavitation to the reaction process is intended to reduce the processing time from $\sim 1-4$ hours needed in batch processing to less than $\sim 30$ seconds. Also, it reduces separation time from $\sim 10$ hours, down to $\sim 1$ hour.

\section{Biodiesel Chemistry}

Biodiesel has several advantages such as: 1) being biodegradable, 2) being non-toxic, 3) having low emissions of carbon monoxide, 4) having a relatively high flash point $\left(150^{\circ} \mathrm{C}\right)$, which makes it less volatile and safer to transport handle than petro-diesel, and 5) it has a good lubricating properties that can reduce engine wear and extend engine life [3]. Transesterification is the most common way to produce biodiesel. It is a catalyzed chemical reaction involving vegetable oil and an alcohol to yield fatty acid alkyl esters (i.e., biodiesel) and glycerol (Fig. 1).

\begin{tabular}{|c|c|c|c|}
\hline \multicolumn{4}{|c|}{ The Biodiesel Reaction } \\
\hline $\begin{array}{l}\mathrm{CH}_{2} O \mathrm{OOR}^{\prime} \\
\stackrel{\mathrm{C}}{\mathrm{C}} \mathrm{H}_{2} O \mathrm{OOR} \\
\stackrel{\mathrm{I}}{\mathrm{C}} \mathrm{H}_{2} O \mathrm{OOR}^{\prime \prime}\end{array}$ & $+3 \mathrm{CH}_{3} \mathrm{OH} \stackrel{\text { Catalyst }}{\longrightarrow}$ & $\begin{array}{l}\mathrm{CH}_{2} \mathrm{OH} \\
\stackrel{\mathrm{C}}{\mathrm{C}} \mathrm{H}_{2} \mathrm{OH} \\
\stackrel{\mathrm{C}}{\mathrm{H}} \mathrm{H}_{2} \mathrm{OH}\end{array}+$ & $\begin{array}{l}\mathbf{R}^{\prime} \mathrm{COOCH}_{3} \\
\mathbf{R}^{\prime \prime} \mathrm{COOCH}_{3} \\
\mathbf{R}^{\prime \prime} \mathrm{COOCH}_{3}\end{array}$ \\
\hline $\begin{array}{l}55 \text { gallons } \\
410 \text { lbs } \\
\text { oil or Fat }\end{array}$ & $\begin{array}{c}6.7 \text { gallons } \\
44 \text { lbs } \\
\text { Methanol }\end{array}$ & $\begin{array}{l}4.3 \text { gallons } \\
42 \text { lbs } \\
\text { Glycerin }\end{array}$ & $\begin{array}{l}57 \text { gallons } \\
412 \text { lbs } \\
\text { Biodiesel }\end{array}$ \\
\hline
\end{tabular}

Fig. 1 - A schematic representation of the transesterification of triglycerides (vegetable oil) with methanol to produce fatty acid methyl esters (biodiesel)

(6)

The Triglycerides are the main component of vegetable oil. It consists of three long chain fatty acids esterified to a glycerol backbone. When the triglycerides react with methanol, the three fatty acid chains are released from the glycerol skeleton and combine with the alcohol to produce fatty acid methyl esters (biodiesel) and glycerol as the by-product. R1, R2, and R3 represent Alkyl groups present in the WVO. The catalyst is $\mathrm{NaOH}$ (lye). In general, a large excess of methanol is used to shift the equilibrium far to the right (Fig.1).

\section{Automated Biodiesel Processor Description}

\section{a. Elimination of the Titration Process}

In order to fully automate the process, the first step is eliminating the titration process which is needed before transesterification. This is because each batch of oil has a slightly different composition. The titration process consists of taking a small sample of WVO, and mixing it with lye, water, Phenolphthalein solution and alcohol in order to determine the $\mathrm{pH}$ level of a sample from each batch of oil and determining the correct lye to methanol ratio that is required for a particular batch. The process itself is not that difficult, but it does require careful measurement and operator knowledge of the process. To eliminate this procedure, a certain volume of $\mathrm{WVO}$, a given volume of $\mathrm{CH}_{-} 3 \mathrm{OH}$ (methanol) and a given weight of $\mathrm{NaOH}$ (lye) are combined to produce 
sodium methoxide which will be used for the transesterification reaction. To obtain the needed amounts, a $25 \%$ the oil volume of methanol was used, and for lye 6.25 grams/liter of oil was used. Typically when an operator performs the titration process on a batch, the batch requires between six and seven grams of lye per liter of oil [4]. If fully reacted using 6.7 gallons of methanol and a potassium hydroxide catalyst, 55 gallons of WVO will produce 4.3 gallons of glycerin and 57 gallons of pure biodiesel.

The Transesterification process chosen for this project consists of three steps to eliminate the titration from the process. It is based on the fact that most oils have a similar composition and therefore standard amounts of chemicals per volume of oil can be used (Fig. 1). Additionally, this method has the benefit of more completely reacting the oil with the sodium methoxide and therefore producing more biodiesel than other methods. When the oil reacts all at once it will reach an equilibrium stage at which point the reaction completely stops, even though more glycerin could be removed from the oil. Reacting the oil in two steps restarts the reaction at the second stage, allowing more glycerin to be split out of the waste vegetable oil. However, a drawback to this method is an increase in processing time. For this project this is acceptable as the length of the process is not a critical factor.

\section{b. Biodiesel Processor}

Fig. 2 illustrates the backside of the automated biodiesel processor. Before the actual reaction begins, it is important to preheat the WVO to reduce the viscosity and evaporate any water existing in the oil from cooking. The next step is to pre-filter the WVO in order to remove any food particles remaining in the oil so that the oil is clean and ready for the chemical reactions. To achieve this step, the WVO was poured through a series of progressively finer filtering screens. The finest filtering screen should be around 50 to 75 microns. After the pre-filtering stage, the oil is ready to be added to the 60 gallon tank for processing.

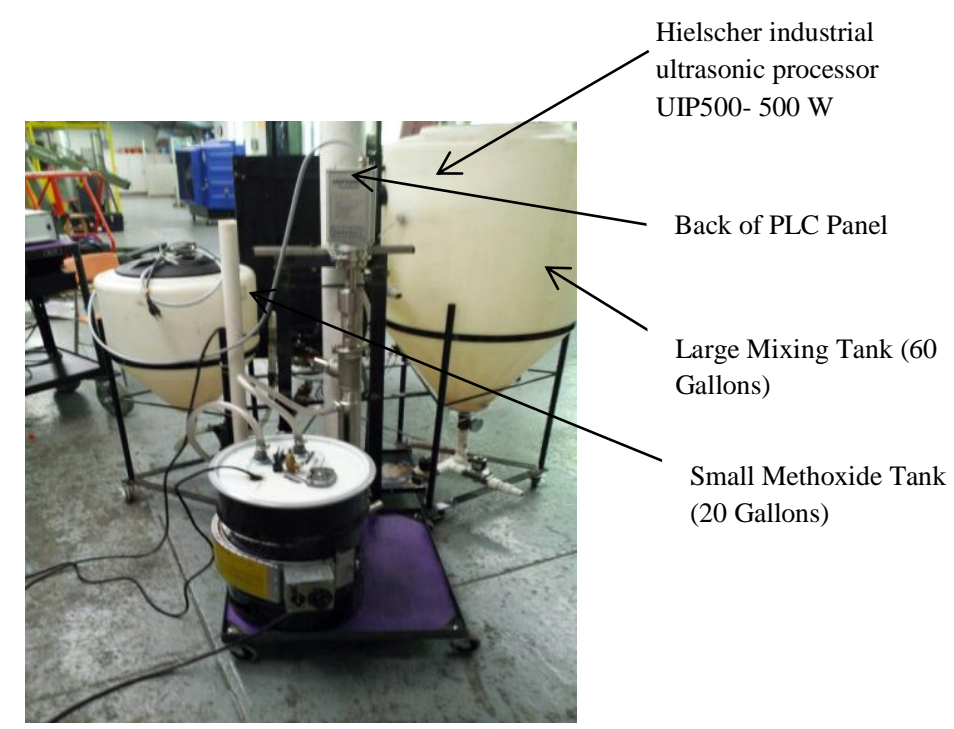

Fig. $2-\quad$ Modified
UltrasonicTransducer $\begin{aligned} & \text { Processor with } \\ & \text { (back of the } \\ & \text { processor with the ultrasonic unit } \\ & \text { assembled) }\end{aligned}$

\section{Ultrasonic Transducer for Mixing WVO}

Mixing WVO is a lengthy process as we have found from prior research. The use of a mixing aid was needed to alleviate some of this time and make a more continuous process. The Hielscher industrial ultrasonic processor UIP500- $500 \mathrm{~W}$ was used for this project. The ultrasonic transducer uses cavitation or nano-sized vacuum bubbles that locally produce high temperatures and extreme pressures when the cavity implodes on itself. This creates jets of liquid that help to overcome the cohesion and adhesion of the WVO and sodium methoxide. This aids in the transesterification and allows for a better and more thorough reaction. Reaction rates can be examined by using a 27/3 test. This test shows how thorough the methanol and WVO have reacted with each other. It is done by mixing 3 parts biodiesel and 27 parts methanol. When mixed together the solution should have a single phase clear look. This signifies a pass. However, if the solution is cloudy and has more than one phase the biodiesel needs to be reacted again (Fig.3). This test correlates well with ASTM equivalent (ASTM D6584) [5]. 


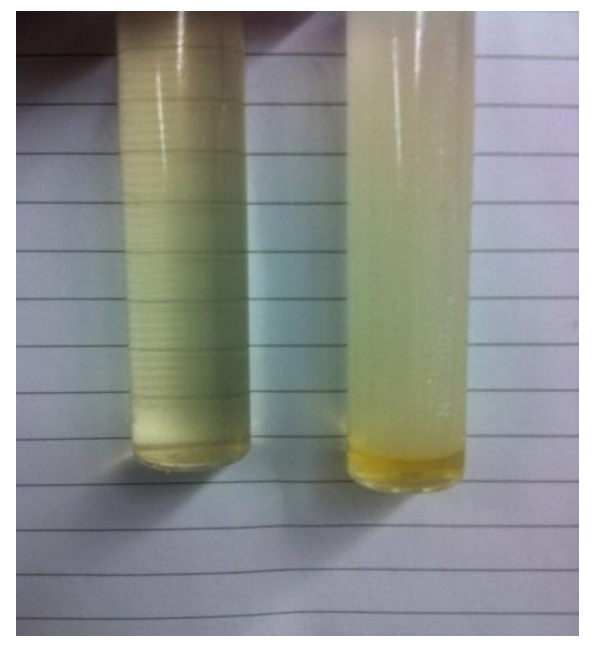

Fig. 3 - A passed 27/3 test using ultrasonication (left) and a failed test using conventional stirring (right).

\section{Biodiesel Filtration and Soap/Glycerol Removal}

In the 20 gallon chemical tank, the methanol and lye were mixed to produce the sodium methoxide. This reaction is exothermic, which produces heat, and results in the formation of sodium methoxide. In the first step, the sodium methoxide was added to the WVO in two stages. First, the oil is reheated between $118^{\circ}-126^{\circ} \mathrm{F}$ $\left(120^{\circ} \mathrm{F}\right.$ is optimum) and $\mathbf{8 0 \%}$ of the sodium methoxide was added. The mixture was maintained at this temperature and mixed for one hour using a circulating pump and the ultrasonic transducer. The mixture is then allowed to cool to room temperature for approximately 2 hours, during which time the glycerin will separate and settle into the bottom of the conical 60-gallon reactor tank where it can be drained off. In the second stage, the partially reacted biodiesel is reheated to an average temperature of $120^{\circ} \mathrm{F}$. The remaining $\mathbf{2 0 \%}$ of sodium methoxide was then added and allowed to circulate/mix for another hour, and then is cooled for another 12 hours before draining any final glycerin that formed.

The final step in the biodiesel process is to remove any soap, water, methanol, and other contaminants from biodiesel that were inadvertently created during the processing.
Traditionally, a wet wash system is used for this process. It requires several wash cycles with water, and each cycle requires $12+$ hours of settling/separation time. Then, it is also common practice to test the $\mathrm{pH}$ of the resulting biodiesel, and add an acid, such as vinegar, as necessary to balance the biodiesel's $\mathrm{pH}$ level. Then the contaminated water must be properly disposed of, adding cost and time to the process.

In this project, a drywash tower was designed specifically for this purpose. Initially the cost is greater, but the return on investment will be much higher because biodiesel can be produced faster and the end product will be of higher quality. Using a dry wash system with ion exchange media keeps us from having to balance the biodiesel $\mathrm{pH}$ by adding in an acid. In this project, a two step dry wash process with Purolite ${ }^{\circledR}$ PD 206 filtering media [6] was planned. Before removing biodiesel from the 60gallon reactor, it must be, or very close to, room temperature. This is because the higher temperatures can damage the filtering media and accelerate the degradation of the PVC pipe. The biodiesel was pumped through the tower at a rate of 7 gallons per hour. It was planned to have two dry wash filtration towers in order to ensure that the maximum amounts of contaminants are removed in a shorter period of time. From the towers, the biodiesel is pumped into a holding air tight and sealed tank, where it is ready to use in virtually any diesel engine.

\section{c. Programmable Logic Controller Programming}

The last step involved is writing a PLC program to control the two phase process and incorporate the drywash towers. The current biodiesel processor uses an Allen Bradley 503 PLC controller. The AB-503 PLC controller was selected because it is currently widely used in industry. It has multiple inputs and output capabilities and is easy to program (Fig. 3). In addition, the PLC will allow the operator to add a vast array of items to the process, such as flow meters, temperature gauges, timers, solenoid valves, and pumps quite easily. The processor works on an input/output basis for each action to occur. Once an input signal/signals are received 
(a given temperature, elapsed time, etc.) the controller sends an output signal (begin mixing, turn heater on, etc.).

The PLC controller has 16 inputs (I:0 - I:15) and 16 outputs on two output cards $(\mathrm{O} 2: 0$ O2:7 and 03:0-O3:7) (Fig. 4). There are three buttons on the PLC panel; a green start button, a red stop button, and a black reset button. The red stop button can be pressed at any time to stop the processor. The reset button can also be used to stop the program, and it must be pressed after the stop button is pressed in order to reset the counters in the program.

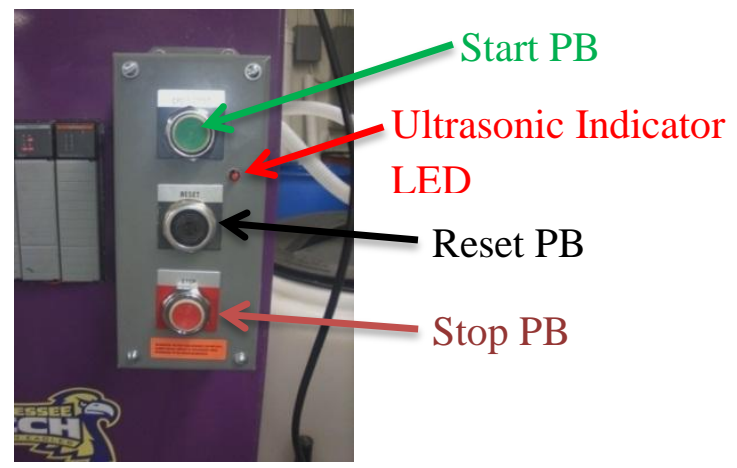

Fig. 3 - Illustrates the 503 Allen Bradley controller

\begin{tabular}{|ll|}
\hline Inputs & \\
I:1/0 & Thermostat \\
I:1/1 & $\begin{array}{l}\text { Fluid Level Sensor } 1 \text { (Bottom of } \\
\text { Small Tank) }\end{array}$ \\
& $\begin{array}{l}\text { Fluid Level Sensor 2 (Top of Large } \\
\text { Tank) }\end{array}$ \\
I:1/3 & $\begin{array}{l}\text { Fluid Level Sensor } 3 \text { (Bottom of } \\
\text { Large Tank) }\end{array}$ \\
I:1/4 & $\begin{array}{l}\text { Stop Button (Red) } \\
\text { I:1/5 }\end{array}$ \\
& $\begin{array}{l}\text { Start Button (Green, bottom button) } \\
\text { (NOTE: Press to start } 1^{\text {st }} \text { and } 2^{\text {nd }}\end{array}$ \\
I:1/6 & $\begin{array}{l}\text { Reset Button (Black, Middle button) } \\
\text { (NOTE: Press and release to stop }\end{array}$ \\
& processor.) \\
Outputs & Pump 2 (Pump 2 for small tank.) \\
O:2/0 & Valve 1 (For circulating small tank) \\
O:2/1 & Valve 2 (For pumping contents of \\
O:2/2 & small tank into large tank) \\
\hline
\end{tabular}

\begin{tabular}{|ll|}
\hline $\mathrm{O}: 2 / 3$ & Valve 3 (For circulating large tank) \\
$\mathrm{O}: 2 / 4$ & $\begin{array}{l}\text { Valve 4 (For final draining of } \\
\text { biodiesel into dry wash towers) }\end{array}$ \\
$\mathrm{O}: 2 / 5$ & Valve 5 (For circulating large tank) \\
$\mathrm{O}: 2 / 6$ & Valve 6 (For glycerin draining) \\
$\mathrm{O}: 2 / 7$ & Pump 1 (On large tank) \\
& \\
$\mathrm{O}: 3 / 0$ & Ultrasonic (For mixing) \\
$\mathrm{O}: 3 / 1$ & LED (Signal Ultrasonic on) \\
$\mathrm{O}: 3 / 7$ & Heater (Inline heater) \\
\hline
\end{tabular}

Fig. 4 - The input/output for the AB 503 PLC

The process happens in two phases. To start, the operator will add the desired amount of WVO oil into the larger 60-gallon tank. For the reactants, the operator will measure out a volume of $22 \%$ methanol for the amount of oil and 6.25 grams of lye for every liter of oil used. During the first phase, the operator will add $80 \%$ of the measured reactants (the remaining $20 \%$ of lye should be kept in an airtight container until it is needed). The methanol and lye will be added to the smaller 20-gallon tank.

Then the operator will press the "Start" button on the PLC panel. This will open valve \#1 on the small 20-gallon tank, valve \#3 and \#5 on the large tank, and turn on both pumps and the heater. Once the oil in the 60 gallon tank reaches $120^{\circ} \mathrm{F}\left(\sim 49^{\circ} \mathrm{C}\right.$ ) (via a thermometer attached to the tank) a 15 minute timer will begin counting. This is to ensure that all of the oil is thoroughly heated. Once the 15 minutes are up, valve \#2 on the small tank will open and begin pumping the sodium methoxide from the small tank into the 60 gallons reactor. Valve \#1 will close 5 seconds later; the large tank will continue to circulate to stir the mixture. Once Fluid Level Sensor \#1 (located in the bottom of the sodium methoxide small tank) reads empty, a 5 second timer will begin counting to make sure the tank is empty before turning off pump \#2. The ultrasonic transducer will then run for one hour as the large tank is circulated.

After one hour, both valves, the ultrasonic transducer, and pump \#1 will shut off. A 1-hour timer will then start. After 1 hour passes, valve \#6 will automatically open to drain glycerin from the 60 gallon reactor. It will close when 
the fluid level sensor \#2 goes false (Sensor \#2 is on the top of the large 60-gallon reactor). The program will now wait for the operator to add in the remaining $20 \%$ of the reactants and press the reset and then the start button. Once the start button is pressed, the processor will carry out the same steps as in phase one.

\section{d. Heating Large Quantities of WVO}

The application of heat is required to speed up the chemical reaction and reducing the oil viscosity as well as remove the remaining water content from the oil. For this system, an inline water heating element was plumbed into the circulation piping. The heating element was a standard inline water heater with 1500 Watts of power and an $\mathrm{AC}$ voltage requirement of 120 Volts. This heater is programmed to regulate with the thermometer sensor. This will fit perfectly with the designed power scheme and PLC program.

\section{Conclusions}

The result of the first phase of the senior project work is an automated processor that is capable of producing biodiesel with very limited operator interaction. For the system operators, a set of complete work instructions have been written to go along with the processor. The produced biodiesel was analyzed by the chemistry department and we believe it does meet the ASTM standards. It was used successfully in running an AMICO diesel engine AD 186FE.

The second phase results are a greatly reduced reaction time of approximately 1 hour using ultrasonication compared with 4 hours by using traditional means. Also, the settling period has been reduced from 12 hours to less than 2 . Small batches were titrated and reacted by traditional means for comparison and 27/3 tests were performed on both these and the ultrasonified fuel. The use of sonochemistry provided B100 of even greater quality with more yield quantities than all previous tests.

\section{Acknowledgements}

This project was funded by the Department Manufacturing and Engineering Technology, The College of Engineering and the Graduate College at Tennessee Technological University. The authors would to acknowledge the contributions of the previous team members: Colt Jackson, John Manier, Karl Nye, Stephen Cole, Stephen Frazier, Caleb Goss, and Justin Wood. Furthermore, would like to acknowledge the efforts of Brent Moore, for working long hours on this project and leading the efforts of his group composed of Will Enicks, Kris Leeper, and Stephen Tischart. Moreover, we would like to thank Van Eric Short of the ECE Department for his help in programming the PLC. Finally, we would like also to thank Mr. Barry Allison and Mrs. Pauline Reyna for proof reading this manuscript.

\section{Bibliography}

[1] A. ElSawy, G. Graham, and M. Baswell, "Automation of Biodiesel Processor from Waste Vegetable Oil using Programmable Logic Controller", 2012 ASEE annual conference, Paper AC 2012-3263, St. Antonio, Texas, June 2012.

[2] http://www.hielscher.com/ultrasonics/sonoc hem_01.htm

[3] http://www.eia.doe.gov/oiaf/analysispaper/b iodiesel/index.html

[4] Y. Zhang, M.A. Dube, D.D. McLean, M. Kates, "Biodiesel production from waste cooking oil: 1. Process design and technological assessment", Elsevier, Bioresource Technology 89 (2003) 1-16.

[5] http://utahbiodieselsupply.com/qualitytests. php

[6] Purolite is a registered trademark of The Purolite Company, 2010. For more information see http://purolite.com/RelId/33637/ISvars/defa ult/Home.htm. 